\title{
Content-Based Image Retrieval: An Assessment
}

\author{
Santosh Kumar Swarnkar1, Prof. Avinash Sharma² \\ 1PG Scholar, ${ }^{2}$ Assistant Professor \\ ${ }^{1,2}$ Department of CSE, MITS, Bhopal, Madhya Pradesh, India
}

\begin{abstract}
How to cite this paper: Santosh Kumar Swarnkar | Prof. Avinash Sharma "Content-Based Image Retrieval: An Assessment" Published in International Journal of Trend in Scientific Research and Development (ijtsrd), ISSN: 24566470, Volume-3 | Issue-3, April 2019, pp.154-156, URL: http://www.ijtsrd.co $\mathrm{m} /$ papers/ijtsrd217

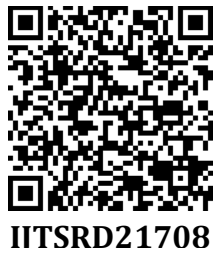
08.pdf

Copyright (C) 2019 by author(s) and International Journal of Trend in Scientific Research and Development Journal. This is an Open Access article distributed under the terms of the Creative Commons

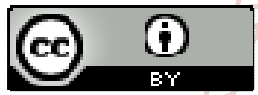
Attribution License (CC BY 4.0) (http://creativecommons.org/licenses/ by $/ 4.0$ )

\section{INTRODUCTION}

There are various processes on web through which one can create, process and store images or any other dimensional information. The outcome has resulted into various studies in searching and managing images. Therefore, finding efficient image retrieval mechanisms from large resources has become a wide area of interest to researchers [1]. Image retrieval method is a technique for searching and retrieving images from a large database of digital images. The need to find the desired image from the image database systems which can be geographical maps, pictures, medical images, pictures in medical atlases, pictures obtaining by cameras, microscopes, telescopes, video cameras, paintings, drawings and architectures plans, drawings of industrial parts, space images, etc. is that it is shared by many professional groups, including journalists, design engineers and art historians [2]

Basically the tricks on images are done with the help of an application (software as a service) locally as well as centrally, by requesting the images stored on the image database. The applications deals with the image storing, image retrieving, image processing and they are: ContentBased Visual Information Retrieval (CBVIR) and ContentBased Image Retrieval (CBIR). Content-based retrieval is an important alternative and it is advantageous over traditional keyword-based searching for multimedia such as image and image data. CBIR can greatly enhance pictorial information management as well as supporting with possible opportunities in the form of statistical and comparative analysis of functional image and image data.

CBIR is a technique for retrieving images on the basis of automatically-derived features such as color, texture and shape. Here a query image will be triggered and will be compared to the images stored in the image database. Once the match is found the results are displayed in the form of image output. The CBIR application extracts the image from the image database with the help of input image or image data. The desired image extracted can be used for various purposes such as communication, analysis, guidelines, etc. CBIR application also characterizes image queries into three levels of abstraction: primitive features such as color or shape, logical features such as the identity of objects shown.

\section{LITERATURE SURVEY}

\subsection{Content-based Image Retrieval Based on Eye- tracking}

Ying Zhou, Jiajun Wang and Zheru Chi in 2018, to improve the performance of an image retrieval system, a novel content-based image retrieval (CBIR) framework with eye tracking data based on an implicit relevance feedback mechanism is proposed in this paper. Our proposed framework consists of three components: feature extraction and selection, visual retrieval, and relevance feedback. First, by using the quantum genetic algorithm and the principle component analysis algorithm, optimal image features with 
70 components are extracted. Second, a finer retrieving procedure based on multiclass support vector machine (SVM) and fuzzy c-mean (FCM) algorithm is implemented for retrieving most relevant images. Finally, a deep neural network is trained to exploit the information of the user regarding the relevance of the returned images. This information is then employed to update the retrieving point for a new round retrieval. Experiments on two databases (Corel and Caltech) show that the performance of CBIR can be significantly improved by using our proposed framework.

\subsection{NIR: Content Based Image Retrieval on Cloud Computing}

Zhuo YANG, Sei-ichiro KAMATA and Alireza AHRARY in 2009 proposed NIR - an open source cloud based content based image retrieval system. CBIR is one of the challenging and emerging technologies as high computation task because of the algorithm computation complexity and big amount of data. As based on cloud computing infrastructure, NIR is easy to extent and flexible for deployment. Due to this there has been significant increase in scalability and availability. As an open source project, NIR can be improved on demand and integrated to other existing systems [3].

\subsection{Content-based Image Retrieval (CBIR) using Hybrid Technique}

This method of CBIR was researched by Zainab Ibrahim Abood, Israa Jameel Muhsin, and Nabeel Jameel Tawfiq in year 2013. The research delivered Content Based Image Retrieval (CBIR) using four feature extraction techniques. The four techniques used are colored histogram features technique, properties features technique, gray level cooccurrence matrix (GLCM) statistical features technique and hybrid technique. The features are extracted from the database images and query images in order to find the similarity measure between them. The similarity-based matching is post extraction stage in CBIR. The three types of similarity measure used are, normalized Mahalanobis distance, Euclidean distance and Manhattan distance. The research concluded that CBIR using hybrid technique have higher match performance in all kind of similarity measures used [4].

\subsection{Content Based Image Retrieval Using Fusion of Gabor Magnitude and Modified Block Truncation Coding}

Dr. H B Kekre, V A. Bharadi have introduced Content Based Image Retrieval using Fusion of Gabor Magnitude and Modified Block Truncation Coding. Gabor filters comprises of wavelets, where each wavelet captures energy at a specific frequency and a specific direction. Expanding a signal using this basis provides a localized frequency description, therefore capturing local features in the form of energy of the signal. Texture features can be extracted from the group of energy distributions. And modified block truncation is used to retrieve color feature from image. The proposed system was giving higher Precision and Recall as compared to only Gabor and only MBTC based CBIR. Gabor feature gives good response to texture of the image and Modified BTC give good response to color content of image [5].

\subsection{Efficient Relevance Feedback for Content-Based Image Retrieval by Mining User Navigation Patterns}

Ja-Hwung Su, Wei-Jyun Huang, Philip S. Yu and Vincent S. Tseng in 2011 proposed a method, Navigation-Pattern-based
Relevance Feedback (NPRF), to achieve the high efficiency and effectiveness of CBIR in coping with the large-scale image data. In terms of efficiency, the iterations of feedback are reduced substantially by using the navigation patterns discovered from the user query log. In terms of effectiveness, the proposed search algorithm NPRF Search makes use of the discovered navigation patterns and three kinds of query refinement strategies, Query Point Movement (QPM), Query Reweighting (QR), and Query Expansion (QEX), to converge the search space toward the user's intention effectively. By using NPRF method, high quality of image retrieval on RF can be achieved in a small number of feedbacks. The experimental results reveal that NPRF outperforms other existing methods significantly in terms of precision, coverage, and number of feedbacks [6].

\subsection{Content Based Image Retrieval Using Independent Component Analysis}

Arti Khaparde, B L Deekshatulu, M.Madhavilatha, Zakira Farheen, Sandhya Kumari presented a new approach for global feature extraction using an emerging technique known as Independent Component Analysis (ICA). A comparative study was delivered between ICA feature vectors and Gabor feature vectors for 180 different texture and natural images in a databank. Result analysis show that extracting color and texture information by ICA provides significantly improved results in terms of retrieval accuracy, computational complexity and storage space of feature vectors as compared to Gabor approaches [7].

\section{DATASET AND PERFORMANCE EVALUATION}

To quantitatively demonstrate the effectiveness and efficiency of various image retrieval algorithms, it is indispensable to collect some benchmark datasets and define the evaluation metrics. In this section, we discuss the recent ground truth datasets and distractor datasets used in experimental study for image retrieval. Besides, we introduce the key evaluation indicators in CBIR, including accuracy, efficiency, and memory cost. 8.1 Recent Dataset for CBIR Intuitively, the ground-truth dataset should be sufficient large so as to well demonstrate the scalability of image retrieval algorithms. However, considering the tedious labor in dataset collection, the existing ground-truth dataset are relatively small, but mixed with random millionscale distractor database for evaluation on scalability. The existing ground-truth datasets target on particular object/scene retrieval or partial-duplicate Web image retrieval. Generally, the ground-truth images contain a specific object or scene and may undergo various changes and be taken under different views or changes in illumination, scale, rotation, partial occlusion, compression rate, etc. Typical ground truth dataset for this task includes the UKBench dataset, the Oxford Building dataset, and the Holidays dataset, etc. MIR Flickr-1M and Flickr-1M are two different millionscale databases which are usually used as distractor to evaluate the scalability of image retrieval algorithms. For convenience of comparison and reference, we list the general information of those recent datasets popularly used in CBIR. UKBench dataset It contains 10,200 images from 2,550 categories 9 . In each category, there are four images taken on the same scene or object from different views or illumination conditions. All the 10,200 images are taken as query and their retrieval performances are averaged. Holidays dataset There are 1,491 images from 500 groups in the Holidays dataset10. Images in each group are taken on a scene or an object with various viewpoints. The 
first image in each group is selected as query for evaluation. Oxford Building dataset (Oxford-5K) The Oxford Buildings Dataset11 consists of 5062 images collected from Flickr12 by searching for particular Oxford landmarks. The collection has been manually annotated to generate a comprehensive ground truth for 11 different landmarks, each represented by 5 possible queries. This gives a set of 55 queries over which an object retrieval system can be evaluated. Some junk images are mixed in it as distractor.

\section{CONCLUSION}

Thus this paper describes how the images will be browsed from local directory, and how they will be stored in a blob storage on cloud. CBIR SaaS architecture is proposed due to which the services of CBIR will be dynamically made available throughout the desired systems resulting in increase in applications scalability, flexibility and availability.

Performance will be evaluated using Similarity measures like precision and recall. The outcome of the similarity measure is expected to be higher than the threshold value. The cloud services provided by cloud architecture will handle all the unexpected traffic, and it will simultaneously benefit with minimized cost. CBIR will no longer behave as a product and hence will be available to the intended users dynamically.

\section{REFERENCES}

[1] Leila Kabbai, Mehrez Abdellaoui and Ali Douik, "Content-based Image Retrieval Based On Eyetracking", https://doi.org/10.1145/3206343.3206353, 2018.

[2] A. Smeulders, M. Worring, S. Santini, A. Gupta and R. Jain (2000), "Content-based image retrieval at the end of the early years", in IEEE Transactions on Pattern Analysis and Machine Intelligence, Vol. 22,1349-1380, December 2000.

[3] Yogita Mistry and D.T. Ingole, "Survey on Content Based Image Retrieval Systems" in International Journal of Innovative Research in Computer and Communication Engineering (IJIRCCE), Vol.1, October 2013.
[4] Zhuo YANG, Sei-ichiro KAMATA and Alireza AHRARY, "NIR: Content Based Image Retrieval on Cloud Computing" in IEEE International Conference on Intelligent Computing and Intelligent Systems, 2009.

[5] Zainab Ibrahim Abood, Israa Jameel Muhsin, Nabeel Jameel Tawfiq, "Content-based Image Retrieval (CBIR) using Hybrid Technique" in International Journal of Computer Applications (0975 - 8887), December 2013.

[6] H B Kekre, V A Bharadi, S D Thepade, B K Mishra, S E Ghosalkar, S M Sawant, "Content Based Image Retrieval Using Fusion of Gabor Magnitude and Modified Block Truncation Coding" in IEEE computer society, 2010.

[7] Ja-Hwung Su, Wei-Jyun Huang, Philip S. Yu and Vincent S. Tseng, "Efficient Relevance Feedback for ContentBased Image Retrieval by Mining User Navigation Patterns", in IEEE Transactions On Knowledge And Data Engineering, Vol. 23, No. 3, 1041-4347/11, March 2011.

[8] Arti Khaparde, B L Deekshatulu, M. Madhavilatha, Zakira Farheen, Sandhya Kumari, "Content Based Image Retrieval Using Independent Component Analysis" in IJCSNS International Journal of Computer Science and Network Security, VOL.8 No.4, April 2008.

[9] S. Selvarajah and S. R. Kodithuwakku "Combined Feature Descriptor for Content Based Image Retrieval", 2011 6th International Conferencon Industrial and Information Systems, ICIIS 2011, Aug. 16-19, 2011, Sri Lanka, IEEE.

[10] M. Arevalillo-Herr'aez, Francesc J. Ferri, Salvador Moreno-Picot, "An interactive evolutionary approach for content based image retrieval", in Proceedings of IEEE International Conference on Systems, Man, and Cybernetics, San Antonio, TX, USA - October 2009.

[11] A R Sawant, V A Bharadi, H B Kekre, Bijith Markarkandy, "Implementation of Content based Image Retrieval and Comparison using Different Distance Measures" in IJAIS - ISSN : 2249-0868 Foundation of Computer Science FCS, New York, USA, (ICWAC) 2013 